\title{
Desenvolvimento de um sistema de resfriamento e conservação de sêmen suíno
}

\author{
[Development of a cooling system and preservation of boar semen] \\ M.N.B. Roner ${ }^{1}$, J.M. Silva Filho ${ }^{2}$, M.S. Palhares ${ }^{2} *$, \\ M. Ziviani ${ }^{3}$, E.F. Nascimento ${ }^{2}$, I.J. Silva ${ }^{2}$ \\ ${ }^{1}$ Médica veterinária autônoma \\ ${ }^{2}$ Escola de Veterinária - UFMG \\ Caixa Postal 567 \\ 30123-970 - Belo Horizonte, MG \\ ${ }^{3}$ Escola de Engenharia - UFMG - Belo Horizonte
}

\begin{abstract}
RESUMO
Um contêiner, com taxa de resfriamento controlada de $37^{\circ} \mathrm{C}$ para temperaturas de 17 ou $5^{\circ} \mathrm{C}$, foi construído para resfriamento e conservação do sêmen suíno. Nele, a taxa de resfriamento inicial (de 37 para $17^{\circ} \mathrm{C}$ ) foi $-0,16^{\circ} \mathrm{C} / \mathrm{min}$ até atingir a temperatura de $17^{\circ} \mathrm{C}$ e $-0,17^{\circ} \mathrm{C} / \mathrm{min}$ até $5^{\circ} \mathrm{C}$. No decorrer do resfriamento, quando da passagem pela temperatura do choque térmico (entre 17 e $8^{\circ} \mathrm{C}$ ), a taxa foi de $-0,0049^{\circ} \mathrm{C} / \mathrm{min}$. A temperatura no interior do contêiner manteve-se estável por 53,58 horas à temperatura de $17 \pm 1^{\circ} \mathrm{C}$, e por 43,32 horas à temperatura de $5 \pm 1^{\circ} \mathrm{C}$.
\end{abstract}

Palavras-chave: suíno, sêmen, transporte de sêmen, resfriamento

\begin{abstract}
A container with controlled cooling rate from $37^{\circ} \mathrm{C}$ to 17 or $5^{\circ} \mathrm{C}$ was constructed for the preservation of boar semen. The container produced a cooling rate initially of $-0.16^{\circ} \mathrm{C} / \mathrm{min}$ for a final temperature of $17^{\circ} \mathrm{C}$ and of $-0.17^{\circ} \mathrm{C} / \mathrm{min}$ for a final temperature of $5^{\circ} \mathrm{C}$. The cooling process, during cold shock temperatures $\left(17^{\circ} \mathrm{C}-8^{\circ} \mathrm{C}\right)$ was of $-0.0049^{\circ} \mathrm{C} / \mathrm{min}$. The container temperature was stable for $53.58 \mathrm{~h}$ at internal temperature of $17 \pm 1^{\circ} \mathrm{C}$ and for $43.32 \mathrm{~h}$ at $5 \pm 1^{\circ} \mathrm{C}$.
\end{abstract}

Keywords: boar, semen, semen transported, cooling

\section{INTRODUÇÃO}

A inseminação artificial (IA) em suínos iniciouse na década de 30, na Rússia, com as pesquisas de Milanov (1934). Porém, o primeiro trabalho prático foi descrito por pesquisadores japoneses quando avaliaram os resultados de fertilidade do sêmen coletado com auxílio de vagina artificial. Desde então, a evolução da IA ocorreu de forma lenta e gradativa. Somente a partir de 1988 observou-se aumento significativo da utilização dessa técnica na espécie suína (Johnson, 1998).

Segundo Cameron (1998), uma das principais expectativas de uso da IA em suínos depende do desenvolvimento de novas técnicas de armazenamento e transporte do sêmen a 15 ou $5^{\circ} \mathrm{C}$. Estudos envolvendo sistemas de transporte de sêmen suíno são, na maioria das vezes, resumidos e traduzem tentativas isoladas de utilização do sêmen transportado.

Recebido em 27 de fevereiro de 2005

Aceito em 22 de setembro de 2005

*Autor para correspondência (corresponding author)

E-mail.palhares@vet.ufmg.br

Apoio: FAPEMIG / CNPq 
Diferentemente do suíno, há vários contêineres descritos na literatura visando a refrigeração, a conservação e o transporte do sêmen eqüino, destacando-se os modelos: Sarstedt (Van der Holt, 1984), Equitainer (Douglas - Hamilton et al., 1984), Celle (Hueck, 1990), MSP-1 e MSP-2 (Silva Filho, 1994) e Palhares (1997). Para o sêmen suíno os pesquisadores russos foram os pioneiros, utilizando o sêmen resfriado para inseminar número razoável de fêmeas (MullerShlosser et al., 1980; Gupalov et al., 1983; Vongpralub et al., 1996).

No Brasil, a utilização do sêmen suíno transportado não é rotina nas centrais de inseminação. Geralmente, cada sistema de produção mantém a sua própria central. Em alguns casos ocorre o transporte das doses inseminantes para centrais próximas e/ou para outros estados (Bortolozzo e Wentz, 1997). No entanto, o transporte é realizado dentro de caixas de isopor, sem nenhum controle da temperatura, e, em alguns casos, em geladeiras de menor tamanho ou caixas térmicas que mantêm temperatura de $17^{\circ} \mathrm{C}$. Essas caixas ou geladeiras portáteis são importadas e encontradas no mercado a um custo muito elevado. $\mathrm{O}$ rigoroso controle sanitário, principalmente nas granjas núcleo, inviabiliza a reentrada das caixas transportadoras, onerando ainda mais o custo de produção.

O objetivo deste estudo foi desenvolver um contêiner para o transporte do sêmen suíno, com taxa controlada de resfriamento e temperatura de manutenção de $17 \pm 1^{\circ} \mathrm{C}$ e $5 \pm 1^{\circ} \mathrm{C}$, por um período mínimo de 24 horas.

\section{MATERIAL E MÉTODOS}

O experimento foi desenvolvido na Escola de Engenharia Mecânica da Universidade Federal Minas Gerais, com a criação de recipiente para transporte e resfriamento de sêmen suíno à temperatura de $17 \pm 1^{\circ} \mathrm{C}$ ou $5 \pm 1^{\circ} \mathrm{C}$.

O contêiner constituiu-se de três blocos de isopor: um bloco compacto de $5 \mathrm{~cm}$ formando o fundo; um bloco central de $22 \mathrm{~cm}$, com perfurações para colocação dos blocos menores, contendo os frascos plásticos que acondicionariam o sêmen diluído a ser resfriado, e uma perfuração central para colocação do sistema refrigerador; e um bloco medindo $6 \mathrm{~cm}$, com função de tampa.

O bloco central era formado por seis blocos menores, com perfurações laterais para colocação das garrafas plásticas, que se movimentavam sobre o bloco central, permitindo atingir a temperatura de $17 \pm 1^{\circ} \mathrm{C}$ e $5 \pm 1^{\circ} \mathrm{C}$.

Os frascos plásticos $^{1}$ utilizados no acondicionamento do sêmen foram colocados dentro dos blocos menores. Esse procedimento foi adotado, para que houvesse a movimentação do bloco menor sobre o bloco central, permitindo atingir as temperaturas de manutenção desejadas. Baseando-se nas curvas realizadas durante a montagem do contêiner em vários préexperimentos, ficou estabelecida a distância de $5,5 \mathrm{~cm}$ do bloco refrigerador, para os blocos menores, para a manutenção da temperatura de $17 \pm 1^{\circ} \mathrm{C}$, e de $1 \mathrm{~cm}$, para a de $5 \pm 1^{\circ} \mathrm{C}$.

Por meio de aberturas laterais, do bloco central e dos blocos menores, houve troca de calor/frio entre os frascos e o sistema refrigerador, obtendo-se taxas de resfriamento controlada. Assim, a perfuração central, na qual abrigava-se o bloco refrigerador, comunicava-se com as paredes laterais por meio de uma abertura de $4 \mathrm{~cm}$, durante as duas primeiras horas, independentemente da temperatura final desejada. Após esse período, os blocos pequenos que continham as doses, nas quais desejava-se uma temperatura de estabilização de $5 \pm 1{ }^{\circ} \mathrm{C}$, foram giradas de forma a permanecer isoladas do bloco refrigerador por $1 \mathrm{~cm}$ de isopor. $\mathrm{O}$ mesmo procedimento foi adotado nos blocos com as doses em que se pretendia manter a temperatura de $17 \pm 1^{\circ} \mathrm{C}$, de forma a proporcionar uma distância de $5,5 \mathrm{~cm}$ do bloco refrigerador, garantida pelo aumento da espessura do isopor nesse ponto.

O sistema refrigerador do contêiner constituiu-se de cilindro fechado nas suas extremidades, confeccionado a partir de uma liga de latão/alumínio. Esse cilindro foi revestido por tela de arame, com a função de manter o contato homogêneo das doses com o sistema refrigerador. $\mathrm{O}$ interior do cilindro foi preenchido com dois litros de água e mantido em

\footnotetext{
${ }^{1}$ Minitub
} 
congelador a $-20^{\circ} \mathrm{C}$, por no mínimo 18 horas, antes da sua utilização.

As curvas de resfriamento foram obtidas a partir de dois contêineres, a $17 \pm 1^{\circ} \mathrm{C}$ e a $5 \pm 1^{\circ} \mathrm{C}$. Para tal, utilizou-se água em garrafas plásticas com capacidade $100 \mathrm{ml}$, mantidas em banho-maria ${ }^{2}$ a $37^{\circ} \mathrm{C}$, até o momento da colocação no contêiner.

As leituras das temperaturas no interior do contêiner foram realizadas pelo Software Tracker Datapaq, versão $4^{3}$. Foram utilizados termopares tipo K, sendo um deles calibrado pelo método de comparação, conforme procedimento NBR$3522 / 95$, com a escala de erro de $\pm 0,2$ estimada para nível de confiança de $95 \%$. Os demais termopares utilizados na leitura das temperaturas foram comparados ao termopar já calibrado, e suas diferenças calculadas para aferição.

Os termopares foram introduzidos em seis garrafas plásticas para cada contêiner, enquanto um, que se manteve suspenso no ar, mensurava a temperatura ambiente. Os sensores foram colocados no interior das garrafas plásticas, em seu terço final, e os termopares acoplados nas placas de aquisição de dados. Duas horas após o fechamento (momento zero) abria-se o contêiner para o ajustamento da posição das garrafas $\left(17 \pm 1^{\circ} \mathrm{C}\right.$ e $\left.5 \pm 1^{\circ} \mathrm{C}\right)$, quando era novamente fechado e vedado, até o final da curva $(8582$ minutos). Ao término da curva, as placas foram acopladas ao computador para leitura das temperaturas.

Para determinar o comportamento da temperatura no interior do contêiner, foram acompanhadas 12 curvas de resfriamento por um período de 140 horas, sendo que seis curvas foram analisadas à temperatura de $17 \pm 1^{\circ} \mathrm{C}$ e as outras seis a $5 \pm 1^{\circ} \mathrm{C}$.

As leituras (temperaturas interna e ambiente) foram realizadas a cada dois minutos durante as primeiras 24 horas e a cada 10 minutos até 140 horas após o início do resfriamento.

Avaliaram-se a influência da temperatura ambiente e do tempo de armazenamento sobre a temperatura no interior dos dois contêineres

\footnotetext{
${ }^{2}$ Fanem

${ }^{3}$ Microsoft $^{\circledR}$
}

$\left(17 \pm 1^{\circ} \mathrm{C}\right.$ e $\left.5 \pm 1^{\circ} \mathrm{C}\right)$, após o início do resfriamento, de acordo com as seguintes curvas: curva de resfriamento, com valores obtidos entre a temperatura inicial e a temperatura mínima atingida, no interior do contêiner (para $17 \pm 1^{\circ} \mathrm{C}$ e $\left.5 \pm 1^{\circ} \mathrm{C}\right)$; b) curva de estabilização, com valores obtidos entre a temperatura mínima atingida e o início do aquecimento; c) curva de aquecimento, com valores obtidos entre a temperatura do final da estabilização até 140 horas; d) curva total, com valores obtidos durante as 140 horas.

Para o cálculo da taxa de resfriamento, utilizouse a fórmula $\mathrm{TR}=\mathrm{Tf}-\mathrm{Ti} / \mathrm{t}$, em que: $\mathrm{TR}=$ taxa de resfriamento, $\mathrm{Tf}=$ temperatura final, $\mathrm{Ti}=$ temperatura inicial e $\mathrm{t}=$ tempo gasto entre $\mathrm{Tf} \mathrm{e}$ Ti. Calcularam-se, para as curvas de resfriamento, os valores de queda de temperatura entre 37 e $17 \pm 1^{\circ} \mathrm{C}$ no contêiner de $17^{\circ} \mathrm{C}$, e entre 37 e $5 \pm 1^{\circ} \mathrm{C}$, no contêiner de $5^{\circ} \mathrm{C}$.

As curvas de resfriamento foram obtidas por meio de análise de regressão (Snedecor e Cocham, 1980), adotando-se o nível de significância de $95 \%(\mathrm{P}<0,05)$.

\section{RESULTADOS E DISCUSSÃO}

O contêiner proposto tem a forma compacta $(33 \mathrm{~cm}$ de altura $\times 35 \mathrm{~cm}$ largura), facilitando $o$ manuseio e o transporte. Constituído de isopor, oferece a vantagem do baixo custo e resistência suficiente para o transporte. Seu peso $(0,9 \mathrm{~kg}-$ vazio e $4,2 \mathrm{~kg}$ - completo), favorece o envio por via aérea, férrea ou rodoviária.

A disposição das doses, no interior do contêiner permite o transporte de doze doses inseminantes de $100 \mathrm{ml}$. A individualização das doses inseminantes, como realizado neste experimento, é importante para a funcionalidade de um contêiner, porque possibilita maior praticidade no momento da inseminação, permitindo a retirada de uma ou mais doses, mantendo as demais sob refrigeração (Palhares, 1997). Em suínos, apenas Cambó et al. (1988) relataram o transporte do sêmen em garrafas individualizadas. Em relação aos eqüinos, Silva Filho et al. (1991) mostraram a preocupação com a manipulação das doses inseminantes fora do contêiner de transporte, enquanto os modelos Celle (Hueck, 1990) e Sarstedt (Van der Holst, 
1984) permitiram a individualização das doses inseminantes.

Os frascos utilizados foram os comumente empregados na inseminação artificial de suínos. Dessa forma, as doses inseminantes ficam prontas para o uso, evitando-se a sua manipulação após a retirada do interior do contêiner.

Na literatura consultada, os trabalhos com suínos não fazem referência ao volume total a ser transportado, ou ao tipo de envasamento utilizado. Entretanto, em trabalhos envolvendo o transporte de sêmen eqüino, a maior capacidade foi relatada para o contêiner MSP-2 (Silva Filho et al., 1991), totalizando $900 \mathrm{ml}$ (6 recipientes de $150 \mathrm{ml}$ ), o que redundaria em apenas nove doses para o sêmen suíno. O grande volume/dose inseminante (80 a 100ml) para o suíno (Johnson, 1998) dificulta a criação de contêineres com queda homogênea e estabilização da temperatura em seu interior. Isso mostrou a necessidade de se ajustar o posicionamento das doses, duas horas após o início do resfriamento, tanto para a temperatura de manutenção de $17 \pm 1^{\circ} \mathrm{C}$ quanto para a de $5 \pm 1^{\circ} \mathrm{C}$.

O contêiner proposto propicia a opção de duas diferentes temperaturas finais para a manutenção do sêmen resfriado suíno $\left(17 \pm 1^{\circ} \mathrm{C}\right.$ ou $\left.5 \pm 1^{\circ} \mathrm{C}\right)$. Isso permite maior flexibilidade no transporte do sêmen que não resiste ao resfriamento a $5^{\circ} \mathrm{C}$.

Quanto à temperatura de manutenção de $17 \pm 1^{\circ} \mathrm{C}$, observou-se a mínima $\left(16,4^{\circ} \mathrm{C}\right)$ aos $124,66 \pm 5,61$ minutos (Tab. 1). Neste experimento, ficou estabelecido que a curva de manutenção deveria englobar a menor temperatura atingida até a sua elevação a $20^{\circ} \mathrm{C}$. Assim, a taxa de resfriamento entre $37^{\circ} \mathrm{C}$ e a temperatura mínima foi $-0,16 \pm 0,015^{\circ} \mathrm{C} /$ minuto, com tempo de estabilização de $3215,60 \pm 1,275$ minutos $\left(53,58\right.$ horas). Para a curva de $5 \pm 1^{\circ} \mathrm{C}$, a temperatura mínima foi atingida aos 906,40 $\pm 70,48$ minutos, a uma taxa de resfriamento entre $17^{\circ} \mathrm{C}$ e a temperatura mínima de $-0,0049 \pm 0,0007^{\circ} \mathrm{C} /$ minuto (Tab. 1). Além disso, a temperatura de manutenção, até $6^{\circ} \mathrm{C}$, permaneceu até $2599,20 \pm 186,51$ minutos (43,32horas).

Tabela 1. Taxas de resfriamento no interior do contêiner, segundo o intervalo dentro da temperaturas de manutenção de $17 \pm 1^{\circ} \mathrm{C}$ e $5 \pm 1^{\circ} \mathrm{C}$

\begin{tabular}{lcc}
\hline Intervalo & $\begin{array}{c}\text { Tempo } \\
(\mathrm{min})\end{array}$ & $\begin{array}{c}\text { Taxa de } \\
\text { resfriamento }\left({ }^{\circ} \mathrm{C}\right)\end{array}$ \\
\hline $17^{\circ} \mathrm{C}$ & & \\
37 a TM* $\left(16,45^{\circ} \mathrm{C}\right)$ & $124,66 \pm 5,609$ & $-0,16 \pm 0,0147 \mathrm{a}$ \\
$\mathrm{TM}$ a $19^{\circ} \mathrm{C}$ & $3215,60 \pm 1,275$ & $-0,0022 \pm 0,0010 \mathrm{~b}$ \\
19 a $24^{\circ} \mathrm{C}$ & $8.645,30 \pm 0,00$ & $0,0007 \pm 0,00009 \mathrm{c}$ \\
$5^{\circ} \mathrm{C}$ & & \\
37 a $17^{\circ} \mathrm{C}$ & $112,40 \pm 14,92$ & $-0,18 \pm 0,02 \mathrm{a}$ \\
17 a $8^{\circ} \mathrm{C}$ & $339,60 \pm 32,44$ & $-0,038 \pm 0,02 \mathrm{~b}$ \\
8 a TM $\left(5,38^{\circ} \mathrm{C}\right)$ & $906,40 \pm 70,48$ & $-0,0049 \pm 0,0007 \mathrm{c}$ \\
$\mathrm{TM}$ a $6^{\circ} \mathrm{C}$ & $2.599,20 \pm 186,51$ & $0,00066 \pm 0,0001 \mathrm{c}$ \\
$6^{\circ} \mathrm{C}$ a $24^{\circ} \mathrm{C}$ & $8.648,00 \pm 0,00$ & $0,0027 \pm 0,00008 \mathrm{c}$ \\
\hline * $\mathrm{TM}=$ temperatura $^{\circ}$ & mínima alcançada no interior do \\
contêiner & \\
Médias seguidas por letras diferentes, na mesma coluna, para \\
$17^{\circ} \mathrm{C}$ e $5^{\circ} \mathrm{C}$ diferem entre si $(\mathrm{P}<0,05)$.
\end{tabular}

O tempo necessário para se alcançar a temperatura mínima de $17^{\circ} \mathrm{C}$ foi 124,66 e 112,40 minutos, respectivamente, para as temperaturas finais de $17 \pm 1^{\circ} \mathrm{C}$ ou $5 \pm 1^{\circ} \mathrm{C}$, respectivamente (Tab. 1; Fig. 1 e 2). Isso equivale ao tempo médio de duas horas de armazenamento. Esses valores foram inferiores àqueles descritos por Nascimento et al. (1998), quando foram necessárias quatro horas para se atingir a temperatura de $16^{\circ} \mathrm{C}$, em um sistema de refrigeração com temperatura controlada. Entretanto, Weber (1989) mostrou que até a temperatura de $15^{\circ} \mathrm{C}$ a taxa de resfriamento não é o fator mais importante na viabilidade espermática. 


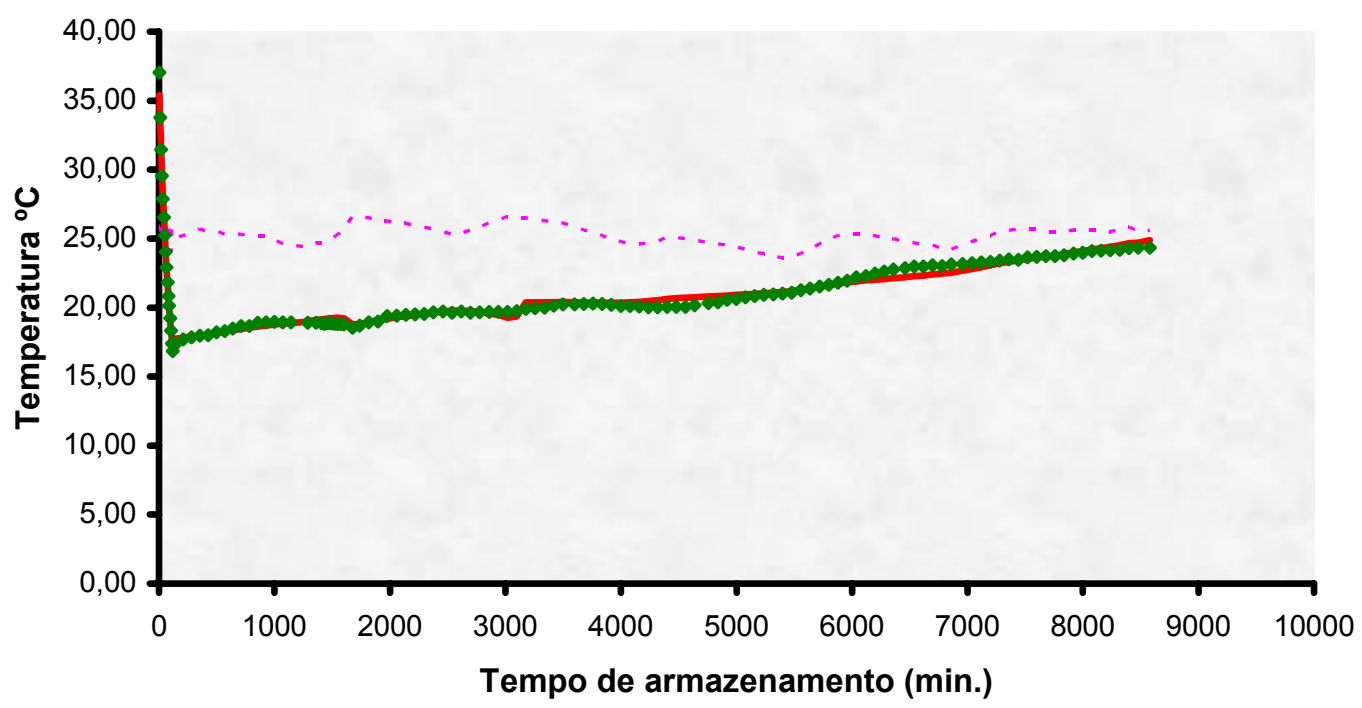

- Resultados experimentais $\longrightarrow$ Modelo teórico - - - - - Temperarura ambiente

Figura 1. Curvas médias da temperatura no interior do contêiner de $17 \pm 1^{\circ} \mathrm{C}$ e a temperatura ambiente, em relação ao tempo de armazenamento

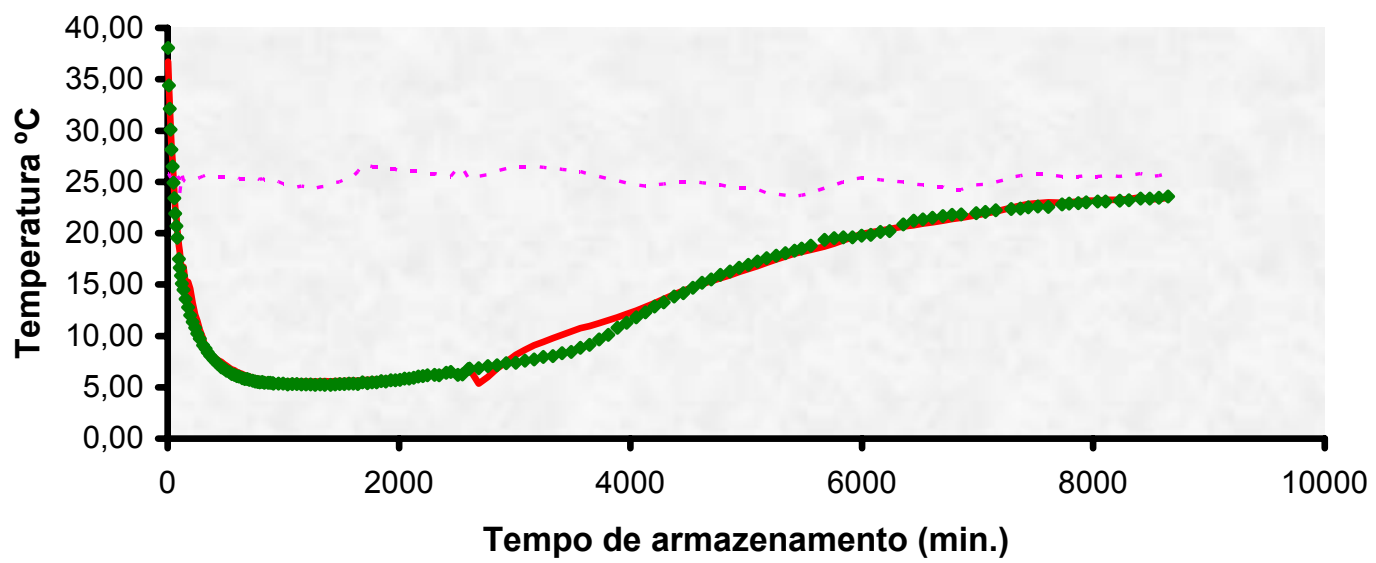

- Resultados experimentais —Modelo teórico - - - - - Temperatura ambiente

Figura 2. Curvas médias da temperatura no interior do contêiner de $5 \pm 1^{\circ} \mathrm{C}$ e a temperatura ambiente, em relação ao tempo de armazenamento.

Para a temperatura final de $5^{\circ} \mathrm{C}$, Nascimento et al. (1998) descreveram um tempo de sete a oito horas. No presente estudo, o tempo gasto foi de $906,40 \pm 70,48$ minutos $(15,11$ horas), a uma taxa de resfriamento de $-0,0049 \pm 0,0007^{\circ} \mathrm{C} /$ minuto.
Essas taxas estão de acordo com o proposto por Kayser et al. (1992), segundo os quais as taxas de resfriamento de $-0,1^{\circ} \mathrm{C} / \mathrm{min}$ e, preferivelmente, de $-0,005$ ou $-0,012^{\circ} \mathrm{C} / \mathrm{min}$, entre 20 e $5^{\circ} \mathrm{C}$, foram superiores às de 
$\geq-0,3^{\circ} \mathrm{C} / \mathrm{min}$, para a manutenção das características de motilidade do sêmen eqüino. $\mathrm{O}$ resfriamento rápido, $\mathrm{o}$ choque térmico $\mathrm{e}$ a centrifugação aumentam a taxa de peroxidação, entretanto, o resfriamento lento e a presença de dióxido de carbono detêm, de maneira reversível, a motilidade, o metabolismo e a peroxidação (Rowson, 1975). A estocagem do sêmen a baixas temperaturas deveria prolongar a viabilidade dos espermatozóides, pela redução dos processos metabólicos (Althouse et al., 1998). Entretanto, as células espermáticas do suíno são bastante sensíveis ao resfriamento e ao congelamento, sendo a sua viabilidade significativamente reduzida a baixas temperaturas (Pursel et al., 1973; Almond, 1994). Dessa forma, a taxa de resfriamento assume um importante papel na manutenção da viabilidade espermática, entre 15 e $5^{\circ} \mathrm{C}$ (Weber, 1998).

$\mathrm{Na}$ construção de um contêiner deve-se dar atenção ao problema de estabilização da temperatura. No presente trabalho, verificou-se a manutenção da temperatura de $17 \pm 1^{\circ} \mathrm{C}$, por 53 horas e a de $5 \pm 1^{\circ} \mathrm{C}$ por 43 horas (Tab. 1; Fig. 1 e $2)$, respectivamente. Os valores encontrados nesse experimento, em relação ao tempo de manutenção, são considerados bons. Segundo Johnson (1998), em muitos países, o tempo gasto para o transporte das doses inseminantes é de no máximo 24 horas. Para o autor, um bom sistema de transporte permite a utilização das doses transportadas (por um, dois e no máximo três dias) para a inseminação das fêmeas sem, no entanto, afetar a sua capacidade fertilizante. Além disso, há que se ressaltar maior tempo de manutenção para a temperatura final de $17 \pm 1^{\circ} \mathrm{C}$ (Fig. 1). Possivelmente, sua maior proximidade com a temperatura ambiente favoreceu a sua manutenção.

Em eqüinos, dentre os sistemas passivos para o resfriamento do sêmen diluído, o modelo Equitainer oferece o maior tempo de manutenção (50-60 horas), enquanto o modelo Celle ultrapassou a temperatura de $8^{\circ} \mathrm{C}$, após 22 horas do início do resfriamento (Hueck, 1990). No Brasil, Palhares (1997) verificou que o tempo de estabilização foi de 1560 minutos, ou seja, 26 horas.

Ao se avaliar a curva de aquecimento, verificouse que aos 3215 minutos a temperatura no interior do contêiner era $19,73 \pm 0,88$, para a curva $17 \pm 1^{\circ} \mathrm{C}$, e aos 2599 minutos, $6,57 \pm 0,58$, para a curva de $5^{\circ} \mathrm{C} \pm 1^{\circ} \mathrm{C}$. Fez-se o acompanhamento da temperatura no interior do contêiner até $24,33 \pm 0,39$ e $23,48 \pm 0,08^{\circ} \mathrm{C}$ para as curvas de 17 e $5^{\circ} \mathrm{C}$, respectivamente, alcançada aos 8582 minutos, ou seja, 143 horas.

As equações de predição da temperatura do sêmen em relação ao tempo de resfriamento e à temperatura ambiente, para as diversas situações, foram:

1. Curva total $-17^{\circ} \mathrm{C}$

Tsêmen $=309,493-0,0003227 \mathrm{~T}+0,0000001188 \mathrm{~T}^{2}-23,3342 \mathrm{TA}+0,4687 \mathrm{TA}^{2}$

$\mathrm{R}^{2}=0,448$

$\mathrm{T}, \mathrm{T}^{2}, \mathrm{TA}, \mathrm{TA}^{2}(\mathrm{P}<0,0001)$

2. Curva total $-5^{\circ} \mathrm{C}$

Tsêmen $=-342,773-0,001626 \mathrm{~T}+0,0000004630 \mathrm{~T}^{2}+28,7296 \mathrm{TA}-0,585087 \mathrm{TA}^{2}$

$\mathrm{R}^{2}=0,644$

$\mathrm{T}, \mathrm{T}^{2}, \mathrm{TA}, \mathrm{TA}^{2}(\mathrm{P}<0,0001)$

3. Curva de resfriamento $-17^{\circ} \mathrm{C}\left(37^{\circ} \mathrm{C}\right.$ a $\left.\mathrm{TM}^{*}\right)$

Tsêmen $=157,124-0,2487 \mathrm{~T}+0,0007706 \mathrm{~T}^{2}-9,7860 \mathrm{TA}+0,1972 \mathrm{TA}^{2}$

$\mathrm{R}^{2}=0,965$

$\mathrm{T}, \mathrm{T}^{2}, \mathrm{TA}, \mathrm{TA}^{2}(\mathrm{P}<0,0001)$

4. Curva de resfriamento $-5^{\circ} \mathrm{C}\left(37^{\circ} \mathrm{C}\right.$ a $\left.17^{\circ} \mathrm{C}\right)$

Tsêmen $=-96,1488-0,299139+0,00110546 \mathrm{~T}^{2}+10,8732 \mathrm{TA}-0,220937 \mathrm{TA}^{2}$

$\mathrm{R}^{2}=0,939$

$\mathrm{T}, \mathrm{T}^{2}(\mathrm{P}<0,0001)$

$\mathrm{TA}, \mathrm{TA}^{2}(\mathrm{P}>0,05)$ 
5. Curva de resfriamento $-5^{\circ} \mathrm{C}\left(17^{\circ} \mathrm{C}\right.$ a $\left.8^{\circ} \mathrm{C}\right)$

Tsêmen $=6430,24-0,0523268 \mathrm{~T}+0,0000507853 \mathrm{~T}^{2}-501,827 \mathrm{TA}+9,82293 \mathrm{TA}^{2}$

$\mathrm{R}^{2}=0,949$

$\mathrm{T}, \mathrm{T}^{2}, \mathrm{TA}, \mathrm{TA}^{2}(\mathrm{P}<0,0001)$

6. Curva de resfriamento $-5^{\circ} \mathrm{C}\left(8^{\circ} \mathrm{C}\right.$ a TM* $)$

Tsêmen $=577,561-0,0145885 \mathrm{~T}+0,00000790913 \mathrm{~T}^{2}-44,9111 \mathrm{TA}+0,892165 \mathrm{TA}^{2}$

$\mathrm{R}^{2}=0,910$

$\mathrm{T}, \mathrm{T}^{2}, \mathrm{TA}, \mathrm{TA}^{2}(\mathrm{P}<0,0001)$

7. Curva de manutenção $-17^{\circ} \mathrm{C}\left(\mathrm{TM}\right.$ a $\left.19,78^{\circ} \mathrm{C}\right)$

Tsêmen $=-123,658+0,001495 \mathrm{~T}-0,0000002381 \mathrm{~T}^{2}+11,31 \mathrm{TA}-0,2265 \mathrm{TA}^{2}$

$\mathrm{R}^{2}=0,416$

$\mathrm{T}, \mathrm{T}^{2}, \mathrm{TA}, \mathrm{TA}^{2}(\mathrm{P}<0,0001)$

8. Curva de manutenção $-5^{\circ} \mathrm{C}\left(\mathrm{TM}\right.$ a $\left.6^{\circ} \mathrm{C}\right)$

Tsêmen $=-17,9777-0,00145992 \mathrm{~T}+0,00000062306 \mathrm{~T}^{2}-0,888231 \mathrm{TA}+0,0169966 \mathrm{TA}^{2}$

$\mathrm{R}^{2}=0,702$

$\mathrm{T}, \mathrm{T}^{2}(\mathrm{P}<0,0001)$

$\mathrm{TA}, \mathrm{TA}^{2}(\mathrm{P}>0,05)$

9. Curva de aquecimento $-17^{\circ} \mathrm{C}\left(19,78^{\circ} \mathrm{C}\right.$ a $\left.24^{\circ} \mathrm{C}\right)$

Tsêmen $=42,3809-0,0002369 \mathrm{~T}+0,00000009454 \mathrm{~T}^{2}-1,9796 \mathrm{TA}+0,04309 \mathrm{TA} \mathrm{A}^{2}$

$\mathrm{R}^{2}=0,911$

$\mathrm{T}, \mathrm{T}^{2}(\mathrm{P}<0,0001)$

TA, $\mathrm{TA}^{2}(\mathrm{P}>0,005)$

10. Curva de aquecimento $-5^{\circ} \mathrm{C}\left(6^{\circ} \mathrm{C}\right.$ a $\left.24^{\circ} \mathrm{C}\right)$

Tsêmen $=137,779+0,00906934 \mathrm{~T}-0,000000535245 \mathrm{~T}^{2}-12,6160 \mathrm{TA}+0,259559 \mathrm{TA}^{2}$

$\mathrm{R}^{2}=0,989$

$\mathrm{T}, \mathrm{T}^{2}, \mathrm{TA}, \mathrm{TA}^{2}(\mathrm{P}<0,0001)$,

em que: Tsêmen $=$ temperatura no interior do contêiner; $\mathrm{T}=$ tempo de resfriamento; $\mathrm{T}^{2}=$ tempo de resfriamento ao quadrado; $\mathrm{TA}=$ temperatura ambiente; $\mathrm{TA}^{2}=$ temperatura ambiente ao quadrado; $\mathrm{R}^{2}=$ coeficiente de correlação, TM=temperatura mínima.

Quando se utilizou a equação de predição, do tempo zero até 8582 minutos, o $\mathrm{R}^{2}$ obtido, tanto para a curva a $17 \pm 1^{\circ} \mathrm{C}(0,448)$ quanto para a de $5 \pm 1{ }^{\circ} \mathrm{C}(0,644)$ foi baixo, resultado da influência de vários fatores sobre o contêiner, destacandose os efeitos do tempo de resfriamento e da temperatura ambiente $(\mathrm{P}<0,0001)$. Isso reflete a característica do resfriamento passivo. Quando o bloco refrigerador atinge a temperatura de equilíbrio no interior do contêiner, ela se mantém até que a temperatura ambiente comece a influenciar o seu aquecimento. Como não há controlador externo para a manutenção da temperatura, como ocorre nos sistemas ativos de resfriamento, a temperatura no interior do contêiner tende se igualar à temperatura ambiente. Isto se dá de forma mais lenta ou mais rápida, dependendo do isolamento térmico do contêiner. Kayser et al. (1992), Moran et al.
(1992) e Palhares (1997) mostraram a interferência da temperatura ambiente sobre as curvas de resfriamento e temperatura final de armazenamento, obtidas por intermédio de sistemas de resfriamento passivo.

Pode-se observar maior controle dos fatores inerentes ao resfriamento, dividindo o processo em períodos de resfriamento $\left(37^{\circ} \mathrm{C}\right.$ à temperatura mínima alcançada), de manutenção $\left(17\right.$ a $19^{\circ} \mathrm{C}$ e de 5 a $6^{\circ} \mathrm{C}$ ) e de aquecimento (da manutenção até 8582 horas após o resfriamento). Esse fato é traduzido pelo aumento do $\mathrm{R}^{2}$ de 0,448 para 0,965 , na curva de resfriamento e para 0,911 na curva de aquecimento, no contêiner a $17^{\circ} \mathrm{C}$. No entanto, para a curva de manutenção, o $\mathrm{R}^{2}$ foi baixo $(0,416)$. Os resultados indicam que $\mathrm{o}$ período de manutenção $\left(17^{\circ} \mathrm{C}\right.$ a $\left.19^{\circ} \mathrm{C}\right)$ sofre interferência da temperatura ambiente e do tempo $(\mathrm{P}<0,05)$, o que não ocorre na curva de $5 \pm 1^{\circ} \mathrm{C}$, na qual somente há interferência do tempo de armazenamento $(\mathrm{P}<0,05)$ sobre o de manutenção.

Em relação ao contêiner de $5 \pm 1^{\circ} \mathrm{C}$, as curvas de resfriamento de 17 a $8^{\circ} \mathrm{C}$ e de $8^{\circ} \mathrm{C}$ a $\mathrm{TM}$ 
apresentaram $\mathrm{R}^{2}$ de 0,949 e 0,910, respectivamente. Observou-se também que, nesses dois momentos, o tempo de resfriamento e a temperatura ambiente influenciaram $(\mathrm{P}<0,05)$ a temperatura no interior dos frascos.

As análises de regressão permitiram comparar o comportamento da temperatura no interior do contêiner com as esperadas no modelo teórico. Observou-se que esse modelo representa corretamente os resultados experimentais (Fig. 1 e 2), sendo observada uma diferença média entre o modelo teórico e os dados experimentais de $0,06^{\circ} \mathrm{C}$ para $17 \pm 1^{\circ} \mathrm{C}$, e de 0,24 para $5 \pm 1^{\circ} \mathrm{C}$. As maiores diferenças foram observadas nos primeiros quatro minutos, na fase inicial de resfriamento.

Segundo Silva Filho (1994), algumas características importantes devem ser exigidas com relação ao meio de transporte utilizado para o sêmen, tais como: isolamento do meio externo, taxa lenta de resfriamento, temperatura de estabilização, proteção contra atrito, estrutura forte, simples e de fácil manuseio.

O sistema de transporte testado neste experimento foi bastante simples e barato, obtendo-se uma taxa de resfriamento lenta, tanto para a curva a $17 \pm 1^{\circ} \mathrm{C}$, quanto para a $5 \pm 1^{\circ} \mathrm{C}$ e tempo de manutenção médio de 53 e 43 horas, respectivamente.

\section{CONCLUSÕES}

O sistema de resfriamento e conservação do sêmen suíno testado foi simples e de baixo custo, e permitiu duas diferentes temperaturas de manutenção $\left(17 \pm 1^{\circ} \mathrm{C}\right.$ e $\left.5 \pm 1^{\circ} \mathrm{C}\right)$, por tempo médio de 53 e 43 horas, respectivamente. Houve influência do tempo de armazenamento e da temperatura ambiente, por se tratar de um sistema de resfriamento passivo. $\mathrm{O}$ desenvolvimento e a utilização da tecnologia de resfriamento, estocagem e transporte do sêmen suíno pode contribuir para o aumento da difusão da inseminação artificial em granjas de menor escala de produção, melhorar o nível genético pela maximização dos próprios varrões nas granjas com central de inseminação, bem como naquelas que adquirem o sêmen de outras centrais.

\section{REFERÊNCIAS BIBLIOGRÁFICAS}

ALMOND, G.W.; BRITT, J.H.; CARR, J. et al. The AI Book: a field and laboratory technician's guide to artificial insemination in swine, 1994. $108 \mathrm{p}$.

ALTHOUSE, G. C.; WILSON, M.E.; KUSSER, C. et al. Characterization of lower temperature storage limitations of fresh-extended porcine semen. Theriogenology, v.50, p.535-543, 1998.

BORTOLOZZO, F.P.; WENTZ, I. Inseminação artificial em suínos no Brasil. Rev. Bras. Reprod. Anim., v.21, p.13-15, 1997.

CAMBÓ, E.; TORO, Y.; ARIAS, T. Efectividade de la insemination artificial com semen porcino transportado em dos concentraciones espermáticas. In: CONGRESS OF INTERNATIONAL PIG VETERINARY SOCIETY, 10., 1988, Rio de Janeiro. Proceedings... Concordia: Embrapa - CNPSA, 1988. p.319.

CAMERON, R.D.A. Porcine reproduction now and in the future. In: CONGRESS OF THE INTERNATIONAL PIG VETERINARY SOCIETY, 15., 1998, Birmingham. Proceedings... Birmingham: IPVS, 1998. p.209.

DOUGLAS- HAMILTON, D.H.; OSO, R.; OSOL, G. et al. A field study of the fertility of transported equine semen. Theriogenology, v.22, p.291-304, 1984.

GUPALOV, N.; VYSOTSKII, N.; NOVIKOV, A. et al. Transport of semen. Svinovodstvo, n.2, p.18-19, 1983.

HUECK, C. Untersuchungen zur Flússigkonservierung von Pfedisperma unter Verwendun Verschiedener Kühlund Transportsysteme - Laboratudie. 1990, 89f. Thesis (DMV)- Tierärztlichen Hoschschule, Hannover.

JOHNSON, L.A. Current developments in swine semen: preservation, artificial insemination and sperm sexing. In: CONGRESS OF THE INTERNATIONAL PIG VETERINARY SOCIETY, 15., 1998, Birmingham. Proceedings... Birmingham:IPVS, 1998. p.225.

KAYSER, J.P.; AMANN, R.P.; SHIDELER, R.K. Effects of linear cooling rate on motion characteristics of stallion spermatozoa. Theriogenology, v.38, p.601-614, 1992. 
MILANOV, V.K.A. Iskustevennoe osemenenieis (Artificial insemination of live stock). Anim. Breed. Abstr., v.2, p.403, 1934.

MORAN, D.M.; JASKO, D.J.; SQUIRE, E.L. et al. Determination of temperature and cooling rate which induce cold shock in stallion spermatozoa. Theriogenology, v.38, p.999-1012, 1992.

MULLER-SHLOSSER, F.; HEYDORN, K.P.; PAUFLER, S. Preliminary investigations on the effect of different types of heat-conserving packaging on fresh boar semen during transfer. Pigs News Inf., v.3, 1982.

NASCIMENTO, E. F.; SILVA FILHO, J.M.; NASCIMENTO, J.K. Efeito dos diluidores e do resfriamento do sêmen a $16^{\circ} \mathrm{C}$ e $5^{\circ} \mathrm{C}$ sobre algumas características espermáticas de varrões. Arq. Bras. Med. Vet. Zootec., v.52, p.141-146, 1998.

PALHARES, M.S. Adequação de um novo contêiner para o transporte do sêmen eqüino diluido e resfriado: I - Características termodinâmicas e funcionais; II - desempenho reprodutivo das éguas inseminadas. 1997. $246 f$. Tese. (Doutorado em Reprodução Animal)Escola de Veterinária, Universidade Federal de Minas Gerais, Belo Horizonte.

PURSEL, V.G.; SCHULMAN, L.L.; JOHNSON, L.A. Effect at the holding time on storage of boar spermatozoa at $5^{\circ}$ C. J. Anim. Sci., v. 37, p. 785-789, 1973.

ROWSON, L.E.A. Prolonged storage of gametes in relation to fertility and progeny characteristics in farm animals. In: BLANDAU,
R.J. Agind gametes their biology and pathology. New York: S. Karger, 1975. p.249-264.

SILVA FILHO, J.M. Aspectos do manejo reprodutivo e do sêmen na inseminação artificial de éguas. 1994. 408f. Tese (Doutorado em Reprodução Animal) - Departamento de Zootecnia, Universidade Federal de Viçosa, MG.

SILVA FILHO, J.M.; FONSECA, F.A.; PALHARES, M.S. et al. Fertilidade do sêmen eqüino transportado. II. Efeito de diluidores na fertilidade de éguas inseminadas com sêmen diluído, resfriando e transportado em container especial. In: CONGRESSO BRASILEIRO DE REPRODUÇÃO ANIMAL, 9., 1991, Belo Horizonte. Anais... Belo Horizonte: CBRA, 1991. p.369.

SNEDECOR, G.W.; COCHRAN, W.G. Statistical methods. Ames: Iowa State University, 1980. $505 \mathrm{p}$.

VAN DER HOLST, W. De huidge en de toekomstige ontwikkeling van de K.I. bij paarden in Nederland. Vlaams Diergeneeskundig Tijdschrift, v.53, p.302-307, 1984.

VONGPRALUB, T.; BUNSIT, K.; SANCHAISURIYA, $\mathrm{P}$. et al. Effect of extenders and containers under rough-road transport on liquid boar semen quality. Pig News Inf., v.20, 1999. (Abstract 1844).

WEBER, H. Zur Kälteschoskempfindlchkeit von Eberspermiem: Einflu $\beta$ von Verdünnermedium, Inkubation und Abkühlrate. 1989. 103f. ThesisTierärztlichen Hoschschule, Hannover. 